DOI: 10.24835/1607-0763-2017-3-120-125

К вопросу о роли лучевых методов

в диагностике локорегионарного рецидива рака толстой кишки (клиническое наблюдение)

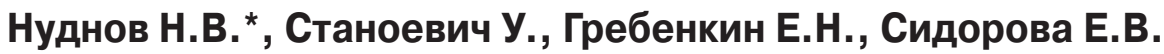

ФГБУ “Российский научный центр рентгенорадиологии” Минздрава России, Москва, Россия

\title{
To the Question of the Role of Radiation Methods in the Diagnosis of Locoregional Recurrence of Colon Cancer (Clinical Case)
}

\author{
Nudnov N.V. ${ }^{*}$, Stanoevich U., Grebenkin E.N., Sidorova E.V. \\ Russian Scientific Center of Roentgenoradiology of the Ministry of Healthcare of the Russian Federation, Moscow, Russia
}

Рак ободочной кишки занимает одно из первых мест в структуре онкологических заболеваний. По данным статистики (под редакцией А.Д. Каприна, В.В. Старинского, Г.В. Петровой) в России за 2015 г. первично зарегистрировано 36494 случая колоректального рака, при этом $2 \%$ случаев не диагностировано. Рецидив рака ободочной кишки может возникнуть на любой стадии независимо от времени, прошедшего после радикального лечения. Локорегионарный рецидив - это наличие опухоли в зоне первичной операции, которая представлена ложем первичной опухоли, анастомозом, брыжейкой кишки с лимфатической системой, брюшиной и соседними органами. Часто после постановки диагноза "рецидив опухоли толстой кишки" больному возможно оказать только паллиативную помощь (наложение колостомы, химиотерапия). Поэтому важно диагностировать рецидив как можно раньше и иметь возможность прогнозировать его возникновение. В статье приводится клиническое наблюдение, подтверждающее важнейшую роль лучевых методов в определении тактики лечения местнораспространенного рака слепой кишки.

Ключевые слова: колоректальный рак, локорегионарный рецидив, илеотрансверзоанастомоз, ирригоскопия, МСКТ.

Ссылка для цитирования: Нуднов Н.В., Станоевич У., Гребенкин Е.Н., Сидорова Е.В. К вопросу о роли лучевых методов в диагностике локорегионарного рецидива рака толстой кишки (клиническое наблюдение). Медицинская визуализация. 2017; 21 (3): 120-125. DOI: 10.24835/1607-0763-2017-3-120-125.

$$
\star \star \star ~
$$

Colon cancer is one of the first places in the structure of oncological diseases. According to statistics, edited by
A.D. Kaprin, V.V. Starinskii, G.V. Petrova of Russia for 2015 was initially 36494 case of colorectal cancer, while $2 \%$ of cases are not diagnosed. Recurrence of colon cancer can occur at any stage regardless of the time elapsed after the radical treatment. Locoregionally originally is the presence of a tumor in the area of primary operation, which is represented by the primary tumor bed, the anastomosis, mesentery of the colon with lymphatic system, peritoneum and adjacent organs. Often after a diagnosis of "recurrence of the tumor in the colon" to the patient it is possible to provide only palliative care (colostomy, chemotherapy). The article cited clinical observation, confirming the important role of radiation techniques in determination of tactics of treatment of locoregional recurrence of the cecum cancer.

Key words: colorectal cancer, locoregional originally, retransliteration barium enema, CT.

Recommended citation: Nudnov N.V., Stanoevich U., Grebenkin E.N., Sidorova E.V. To the Question of the Role of Radiation Methods in the Diagnosis of Locoregional Recurrence of Colon Cancer (Clinical Case). Medical visualization. 2017; 21 (3): 120-125.

DOI: 10.24835/1607-0763-2017-3-120-125.

$$
\star \star \star
$$

\section{Введение}

Выбор тактики лечения колоректального рака в настоящее время невозможен без применения современных методов лучевой диагностики [1-3]. Это в полной мере относится и к диагностике первичной опухоли толстой кишки, особенно при местнораспространенном опухолевом процессе, 
и к диагностике рецидивов [4, 5]. Сложность данной проблемы обусловлена как методическими трудностями выполнения рентгенологических исследований в их классическом варианте, так и сложностями в интерпретации полученной информации в условиях измененных анатомических структур [6, 7].

Приводим собственное клиническое наблюдение.

Пациент К., 70 лет, госпитализирован в ФГБУ "РНЦРР" Минздрава России осенью 2013 г. с клинической картиной острой кишечной непроходимости. При поступлении в РНЦРР у пациента определялся опухолевый узел, занимающий всю правую половину живота, размерами $20 \times 25$ см и прорастающий кожу передней брюшной стенки в нескольких местах (рис. 1).

Клиническая картина заболевания предполагала выполнение хирургического лечения в объеме формирования обходного анастомоза. Для определения распространенности опухолевого процесса в брюшной полости, вовлеченности петель толстой и тонкой кишки, а также других органов и структур больному по экстренным показаниям была выполнена МСКТ органов брюшной полости и грудной клетки с внутривенным контрастированием. По данным МСКТ в правой подвздошной области определялась больших размеров опухоль, исходящая из слепой кишки с инфильтрацией стенок восходящей и терминального отдела подвздошной кишки. Кроме того, опухоль инфильтрировала всю переднюю брюшную стенку, а в правом мезогастрии и правой подвздошной области прорастала переднюю брюшную стенку, выходя на кожу. Несмотря на такое визуальное распространение опухоли, проведенное исследование позволило определить отсутствие прорастания крупных магистральных сосудов, мочеточника и других структур (рис. 2, 3).

Отсутствие прорастания опухолью жизненно важных структур позволило провести пациенту хирургическое лечение в объеме расширенной правосторонней геми-

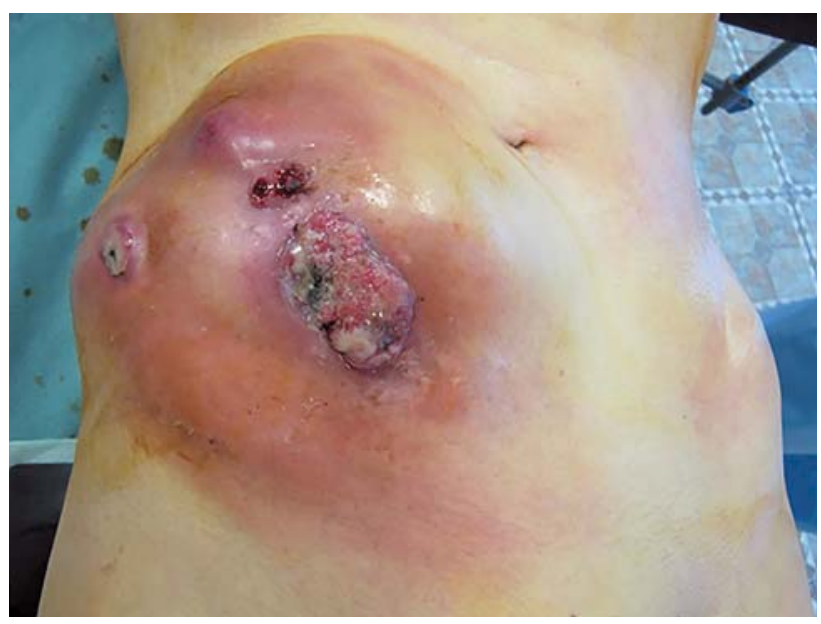

Рис. 1. Опухоль слепой кишки с прорастанием в переднюю брюшную стенку (внешний вид до операции).

колэктомии с обширной резекцией передней брюшной стенки пластикой местными тканями (рис. 4).

Гистологическое исследование: умеренно-дифференцированная аденокарцинома с прорастанием серозной оболочки сигмовидной кишки и врастанием опухоли в переднюю брюшную стенку с образованием в ней полости распада. В 11 из 26 удаленных лимфатических узлов брыжейки были выявлены метастазы аденокарциномы.

От проведения какого-либо варианта дополнительного лечения пациент отказался и с 2014 г. не обследовался. В марте 2017 г. с явлениями кишечного кровотечения экстренно госпитализирован в хирургическое отделение городской больницы. По данным колоноскопии на уровне средней трети поперечной ободочной кишки определяется илеотрансверзоанастомоз “бок в бок" с разрастаниями опухолевой ткани. Опухоль прикрыта сгустками крови, в связи с чем биопсия не проводилась. На фоне проводимой консервативной терапии кровотечение остановлено. Больной самостоятельно выписался из больницы и обратился в "РНЦРР".

Для корреспонденции*: Нуднов Николай Васильевич - 117997 Москва, Профсоюзная ул., д. 86, ФГБУ “РНЦРР" Минздрава России. Тел.: 8-985-224-04-68. E-mail: nudnov@rncrr.ru

Нуднов Николай Васильевич - доктор мед. наук, профессор, заместитель директора по научной работе ФГБУ “РНЦРР” МЗ РФ, Москва; Станоевич Углеша - доктор мед. наук, старший научный сотрудник научно-исследовательского отдела хирургии и хирургических технологий в онкологии ФГБУ “РНЦРР" МЗ РФ, Москва; Гребенкин Егор Николаевич - канд. мед. наук, научный сотрудник научноисследовательского отдела хирургии и хирургических технологий в онкологии ФГБУ "РНЦРР" МЗ РФ, Москва; Сидорова Елена Владимировна - клинический ординатор по рентгенологии ФГБУ “РНЦРР” МЗ РФ, Москва.

Contact*: Nikolay V. Nudnov - Moscow, 117997, Profsoyuznaya str., 86, "RSCRR" Ministry of healthcare of Russia. Phone: 8-985-224-04-68. E-mail: nudnov@rncrr.ru

Nikolay V. Nudnov - doct. of med. sci., professor, deputy Director on scientific work of "RSCRR" Ministry of healthcare of Russia, Moscow; Uglesha Stanoevich - doct. of med. sci., senior researcher of the research Department of surgery and surgical techniques in oncology of "RSCRR" Ministry of healthcare of Russia, Moscow; Egor N. Grebenkin - cand. of med. sci., researcher of the research Department of surgery and surgical techniques in oncology of "RSCRR" Ministry of healthcare of Russia, Moscow; Elena V. Sidorova - a clinical resident in radiology of "RSCRR" Ministry of healthcare of Russia, Moscow. 

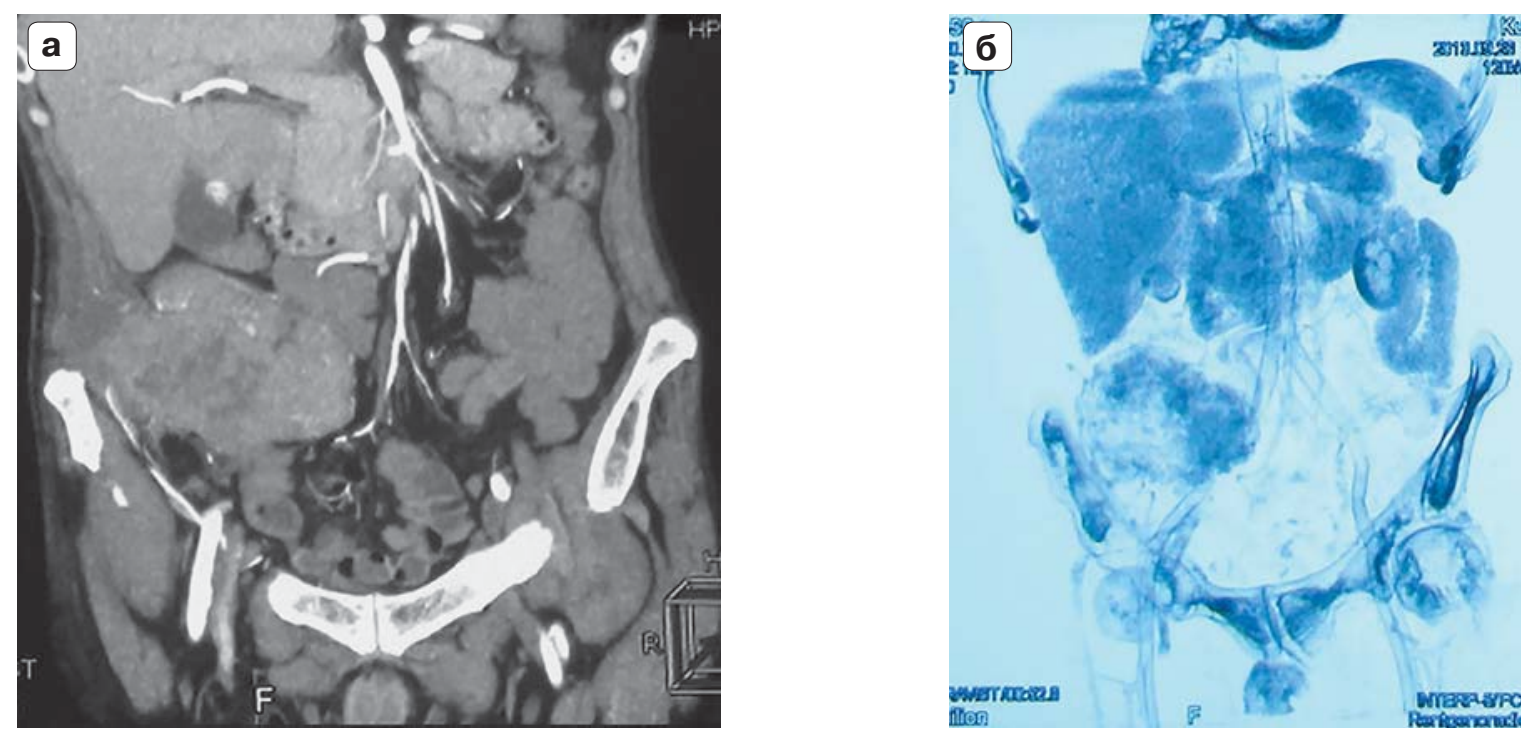

Рис. 2. МСКТ-изображения органов брюшной полости с внутривенным контрастным усилением, фронтальная плоскость. а - опухоль слепой кишки без распространения на верхнюю мезентериальную артерию; б - на подвздошные сосуды.

Рис. 3. МСКТ-изображение органов брюшной полости с внутривенным контрастным усилением, сагиттальная плоскость. Опухоль слепой кишки. Распространения опухоли на печень и правую почку не определяется.

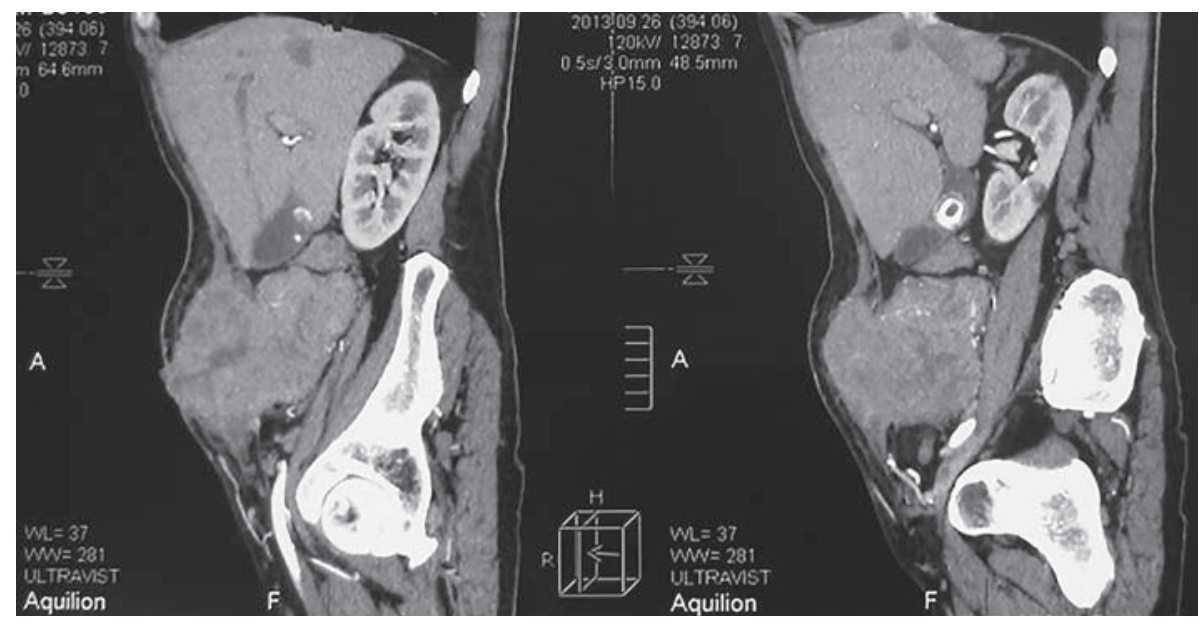

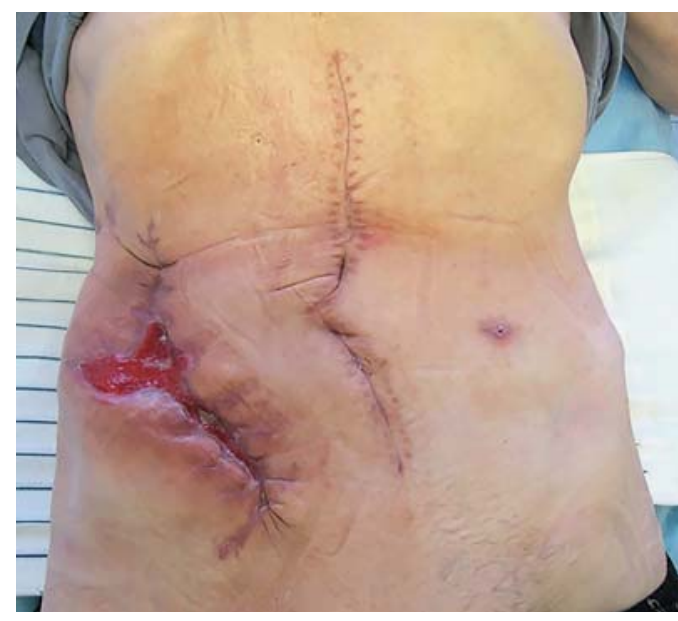

Рис. 4. Послеоперационный рубец через 57 дней после операции (внешний вид). Послеоперационная рана в правом мезогастрии зажила вторичным натяжением.

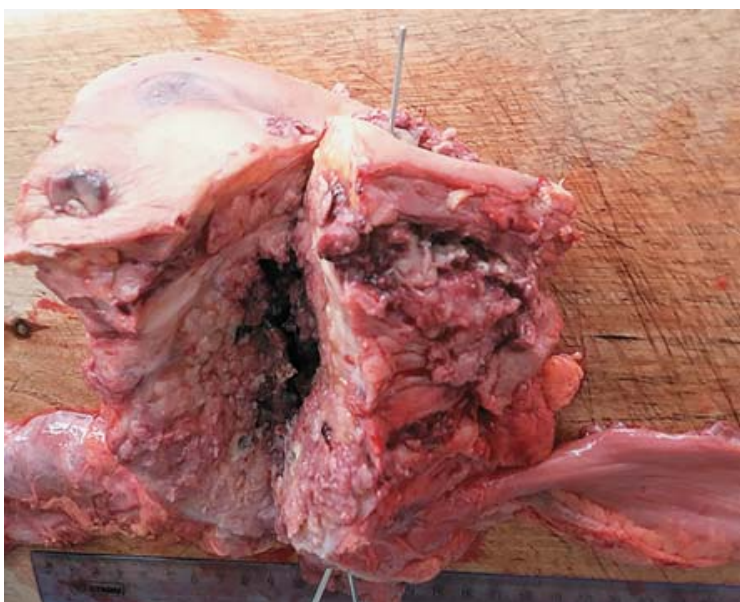

Рис. 5. Послеоперационный макропрепарат. 


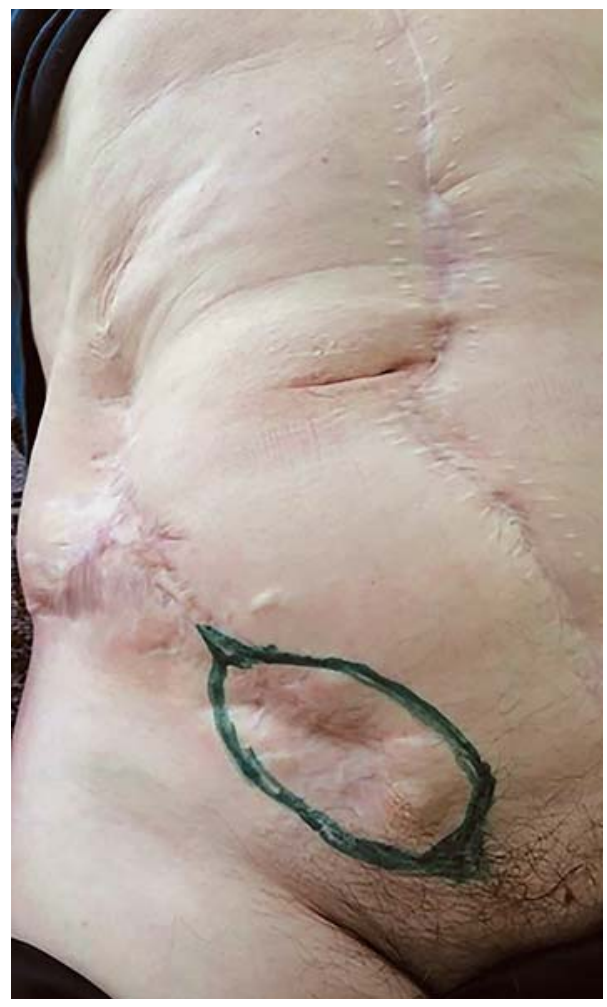

Рис. 6. Внешний вид послеоперационного рубца передней брюшной стенки.

При поступлении жалобы на боли в правой половине живота, периодически возникающее вздутие, наличие плотного образования в рубце передней брюшной стенки правой подвздошной области.

При осмотре послеоперационный рубец после срединой лапаротомии без особенностей. В нижней части послеоперационного рубца в подкожной клетчатке правой подвздошной области пальпируется 3 плотных образования размерами $2 \times 2,1 \times 1$ и $1 \times 0,8$ см. Образования плотно прилежат к апоневрозу (рис. 6, 7).

По данным ирригоскопии в условиях "двойного контрастирования" отмечено равномерное заполнение толстой кишки бариевой взвесью на всем протяжении, вплоть до илеотрансверзоанастомоза. Каких-либо дефектов наполнения, а также сужений или расширений со стороны обследованных отделов толстой кишки не выявлено. Отмечается также заполнение и начальных петель подвздошной кишки, несущей анастомоз. Область анастомоза четко не визуализируется за счет неравномерного дефекта наполнения, утолщения стенок кишок, сужения анастомоза, скопления бариевой взвеси в "слепых карманах" анастомозированных петель кишок (по типу “бок в бок") (рис. 8).

При МСКт с пероральным и внутривенным контрастированием в правой половине брюшной полости выявляется илеотрансверзоанастомоз, стенки которого

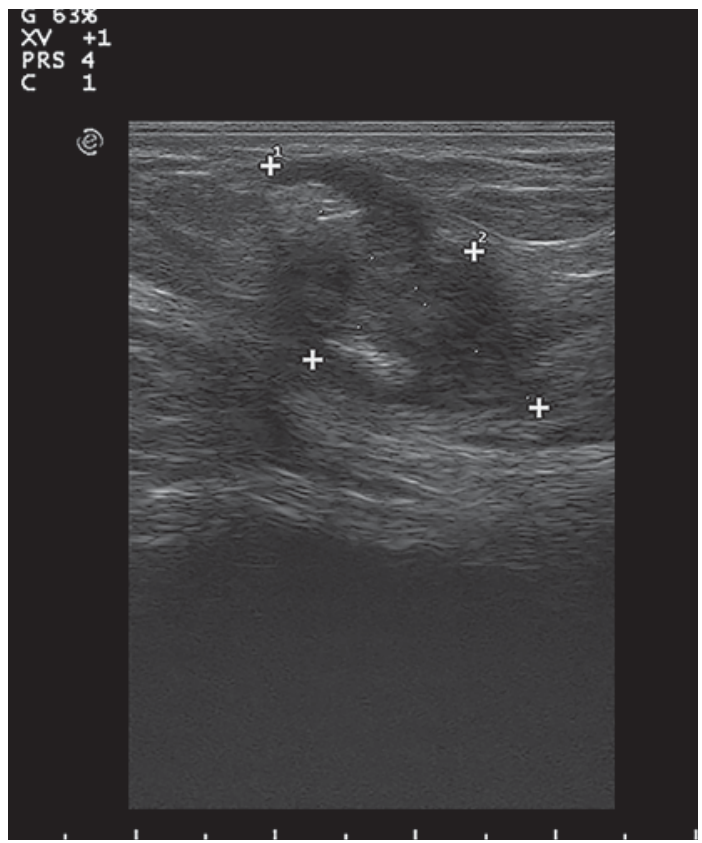

Рис. 7. УЗ-изображение образования мягких тканей правой подвздошной области.

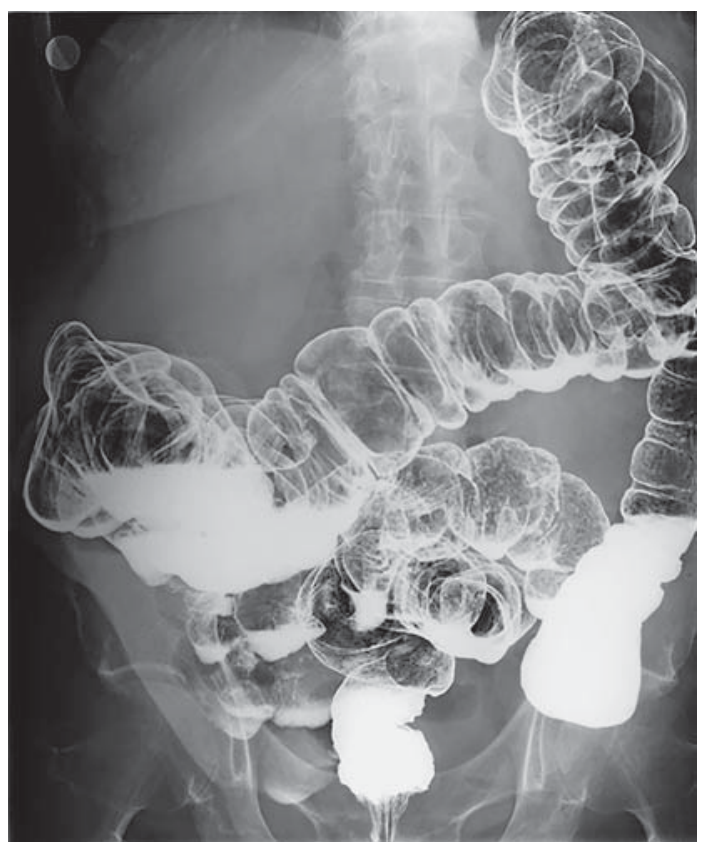

Рис. 8. Ирригограмма в условиях "двойного контрастирования". Косая проекция. Опухоль илеотрансверзоанастомоза.

неравномерно утолщены за счет опухолевой инфильтрации (рис. 9).

На основании результатов обследования больному поставлен следующий основной диагноз: рак слепой кишки рT4bN2M0. Хирургическое лечение в 2013 г. Прогрессирование, рецидив в области илеотрансверзоанастомоза. В плановом порядке выполнено хирурги- 

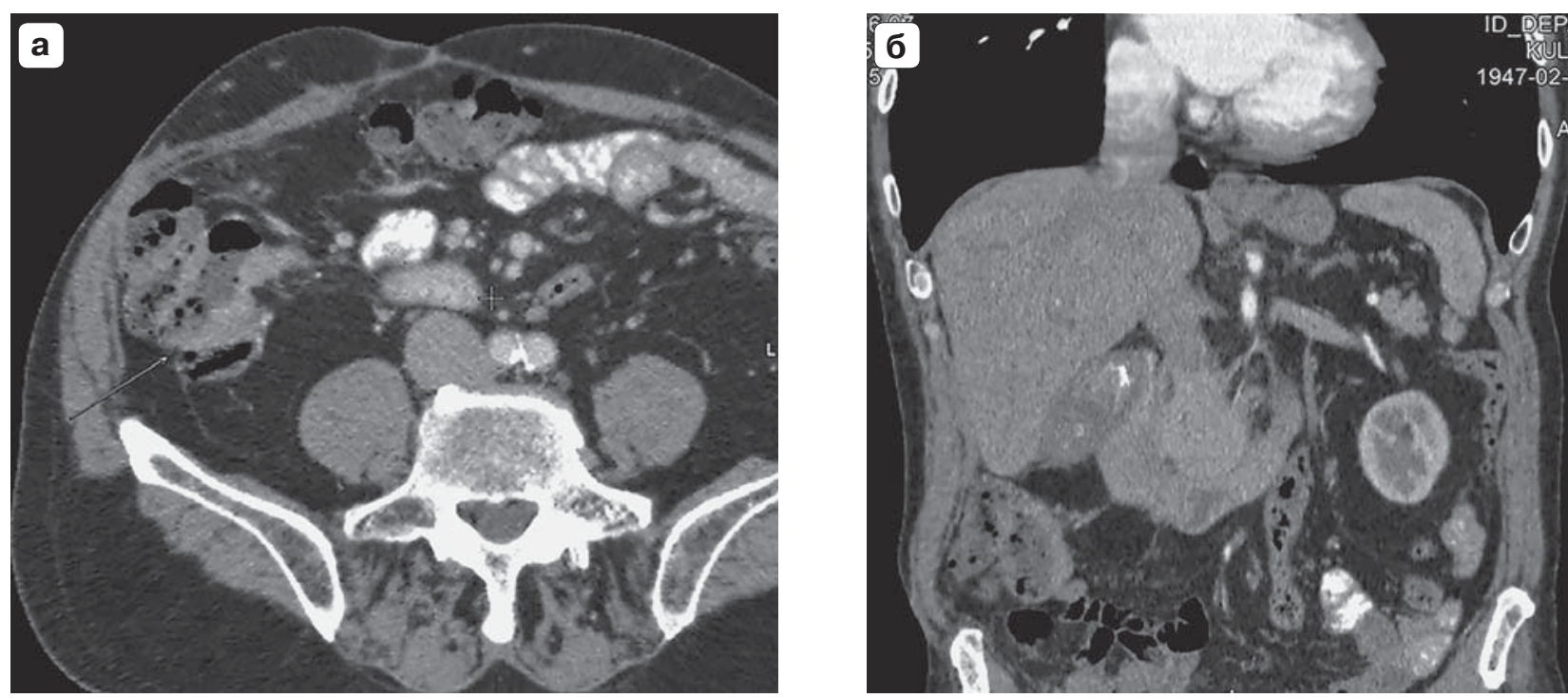

Рис. 9. МСКТ-изображения с пероральным и внутривенным контрастированием. а - аксиальная плоскость; б - фронтальная плоскость. В правой половине брюшной полости выявляются анастомозированные петли поперченой ободочной и подвздошной кишки. Стенки в месте анастомоза неравномерно утолщены за счет опухолевой инфильтрации.

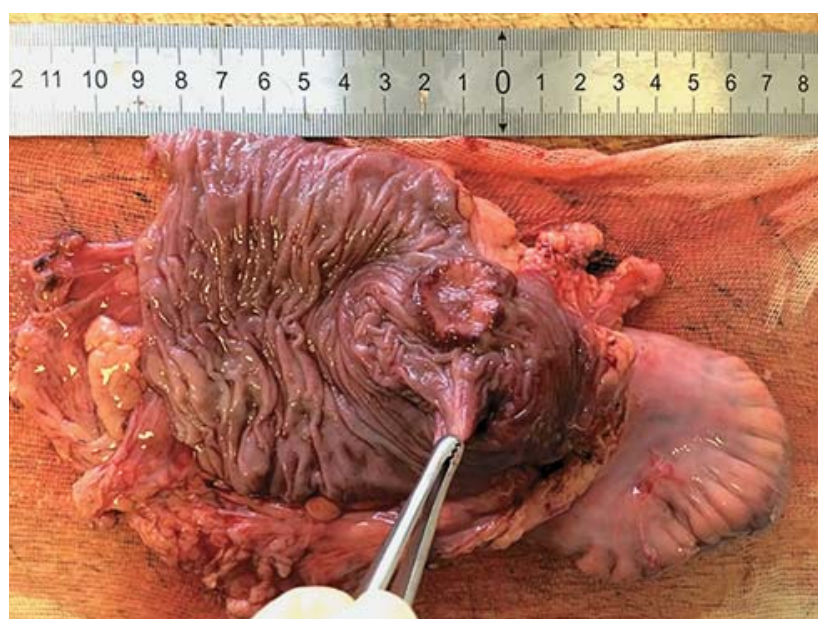

Рис. 10. Вид послеоперационного препарата после проведенного хирургического лечения.

ческое вмешательства в объеме резекции илеотрансверзоанастомоза, иссечение метастазов передней брюшной стенки (рис. 10).

При гистологическом исследовании удаленного препарата: высокодифференцированная тубулярная аденокарцинома толсто-тонкокишечного анастомоза. Опухоль врастает в клетчатку брыжейки толстой кишки без прорастания серозной оболочки. В 4 лимфатических узлах клетчатки брыжейки толстой кишки опухолевые клетки не обнаружены. Во всех трех очагах подкожной жировой клетчатки - узел аденокарциномы толстой кишки.
Данное клиническое наблюдение показывает взаимосвязь лучевых методов диагностики с возможностями радикальной операции при местнораспространенном опухолевом процессе, а также при рецидиве опухоли.

\section{Заключение}

Лучевые методы диагностики наряду с эндоскопическими методами продолжают играть огромную роль как в первичной диагностике опухолевых поражений толстой кишки, так и при рецидиве заболевания. В частности, при ирригоскопии с тугим наполнением и в условиях "двойного контрастирования" удается оценить состояние всех отделов толстой кишки, наличие в них каких-либо дополнительных изменений, сужений, расширений; состояние анастомозированных петель кишок и проходимость анастомоза. Применение МСКТ в сочетании с пероральным и, главное, с внутривенным контрастированием дает дополнительную информацию об утолщении стенки кишки, обусловленном опухолевой инфильтрацией, а также позволяет увидеть распространение процесса за пределы кишки и вовлечение в процесс соседних органов и структур, наличие одиночных или множественных метастатических лимфатических узлов. Все это в конечном итоге определяет тактику лечения больных как с местнораспространенными опухолевым поражением толстой кишки, так и с местными рецидивами онкологических заболеваний данной локализации. 


\section{Список литературы}

1. Соколов Ю.Н., Антонович В.Б. Рентгенодиагностика опухолей пищеварительного тракта. М.: Медицина, 1981: 264-289.

2. Портной Л.М., Сташук Г.А. Современная лучевая диагностика опухолей толстой кишки. Медицинская визуализация. 2000; 4: 4-19.

3. Колганова И.П., Ломовцева К.Х., Кармазановский Г.Г., Калинин Д.В. Диагностика рака толстой кишки. Все ли решено? (Обзор литературы и клинические наблюдения). Медицинская визуализация. 2014; 1: 53-68.

4. Гафуров М.С., Тухбатуллин М.Г. Возможности компьютерной томографии с болюсным контрастированием в диагностике рака толстой кишки и ее осложнений. Онкология. 2005; 2 (11): 32-33.

5. Бутенко А.В., Ахметшина В.М. Локорегионарные рецидивы рака ободочной кишки: проблема, механизмы и лечение. Практическая медицина. 2013; 2 (67): 101-104.

6. Шнигер Н.У. Рентгенодиагностика опухолей прямой и толстой кишок. М.: Медицина, 1973; 235-253.

7. Зароднюк И.В., Тихонов А.А., Орлова Л.П., Жученко А.П., Маркова Е.В. Возможности МСКТ в оценке местного распространения рака ободочной кишки. Медицинская визуализация. 2010; 6: 32-40.

\section{References}

1. Sokolov Yu.N., Antonovich V.B. X-ray diagnostics of tumors of the digestive tract. M.: Medicine, 1981: 264-289. (In Russian)

2. Portnoi L.M., Stashuk G.A. Contemporary Radiology Diagnosis of Colon Tumors. Medical Visualizacion. 2000; 4: 4-19. (In Russian)

3. Kolganova I.P., Lomovtseva K.Kh., Karmazanovsky G.G., Kalinin D.V. Diagnosis of Colon Cancer. Are all the Issues Resolved? (Review of Literature and Clinical Observations). Medical Visualizacion. 2014; 1: 53-68. (In Russian)

4. Gafurov M.S., Tukhbatullin M.G. Possibilities of computer tomography with bolus contrast enhancement in the diagnosis of colon cancer and its complications. Oncologiya. 2005; 2 (11): 32-33. (In Russian)

5. Butenko A.V., Akhmetshina V.M. Locoregional recurrence of colon cancer: the problem, mechanisms and treatment. Practical medicine. 2013; 2 (67): 101-104. (In Russian)

6. Shnigir N.U. Radiological diagnosis of tumors of the colon. M.: Medicine, 1973: 235-253. (In Russian)

7. Zarodnyuk I.V., Tikhonov A.A., Orlova L.P., Zhuchenko A.P., Markova E.V. Value of spiral CT for assessment of local spread of colon cancer. Medical Visualizacion. 2010; 6: 32-40. (In Russian) 